\title{
Microwave-assisted synthesis of some novel 1,4-dihydropyrimidine derivatives of biological interest
}

\author{
Priyanka Pathak, Ramandeep Kaur, and Balbir Kaur* \\ Department of Chemistry, Punjabi University, Patiala 147 002, Punjab, India \\ E-mail : aries_balbir@yahoo.co.in
}

\begin{abstract}
Dihydropyrimidines are associated with broad spectrum of biological activities. In view of this 5-benzoyl-6-methyl-4-substituted phenyl-2-thioxo-1,2,3,4-tetrahydropyrimidines were prepared under microwave radiations, and these tetrahydropyrimidines were then converted to S-alkyl-1,4dihydropyrimidines. Synthesised compounds have been tested for anti-hypertensive activity and have shown some new results about structure-activity relationship.
\end{abstract}

Keywords: 1,4-Dihydropyrimidine, Antihypertensive compounds, aza-analogs of nifedipine, Microwave assisted synthesis

\section{Introduction}

Dihydropyrimidines represent a heterocyclic system with remarkable pharmacological efficiency and are described as potent mimics of dihydropyridine calcium channel blockers ${ }^{1-4}$ e.g. Nifedipine (1). These compounds were first introduced into clinical medicine in 1975. Even today these medicines are used for the treatment of cardiovascular diseases. ${ }^{5}$ Dihydropyrimidines of type 2 also showed similar activity with some new results when properly modified. ${ }^{6-8,12}$ 
<smiles>[R]OC(=O)C1=C(C)NC(C)=C(C(=O)O[R])C1c1ccccc1</smiles>

1<smiles>[X]c1ccccc1C1NC(=[Y])NC(C)=C1C(=O)O</smiles>

2

$\mathrm{Y}=\mathrm{O}, \mathrm{S}$

\section{Results and Discussion}

As a correlation has been observed between the pharmacological activity of this class of calcium channel antagonist and the magnitude at 1,4-dihydro ring puckering. The observed ring distortions were found to be influenced to a great extent by the position of substituent in the 4phenyl ring and the inter ring bond. ${ }^{9}$ In receptor bound conformation ${ }^{10}$ it has been presented that the changes on right hand side(R.H.S.) of the molecule do not affect the activity and hence the substitution on the right hand side was termed non-essential. But contrary to the earlier ${ }^{10}$ report it has been proved by our laboratory ${ }^{11,13}$ that one cannot ignore the structural details at the righthand side also. These observations have increased our interest to synthesize some dihydropyrimidines 4 that can act as valuable substitutes for nifedipine. The changes were made in the substituents at position 2 and 5 of the pyrimidine ring.

The acid catalysed condensation of aromatic aldehyde, benzoyl acetone and thiourea was carried out in an open borosil glassbeaker. Ethanol was used as energy transfer media and the reaction mixture was irradiated in a domestic microwave oven for 4 to 5 minutes. The reaction conditions were optimized. The reaction was followed by TLC, and maximum yield was obtained at 30\% microwave power level. Scheme - 1 shows the synthetic methodology leading towards novel types of conformationally restricted dihydropyrimidine derivatives of Table-1 and Table-2. 


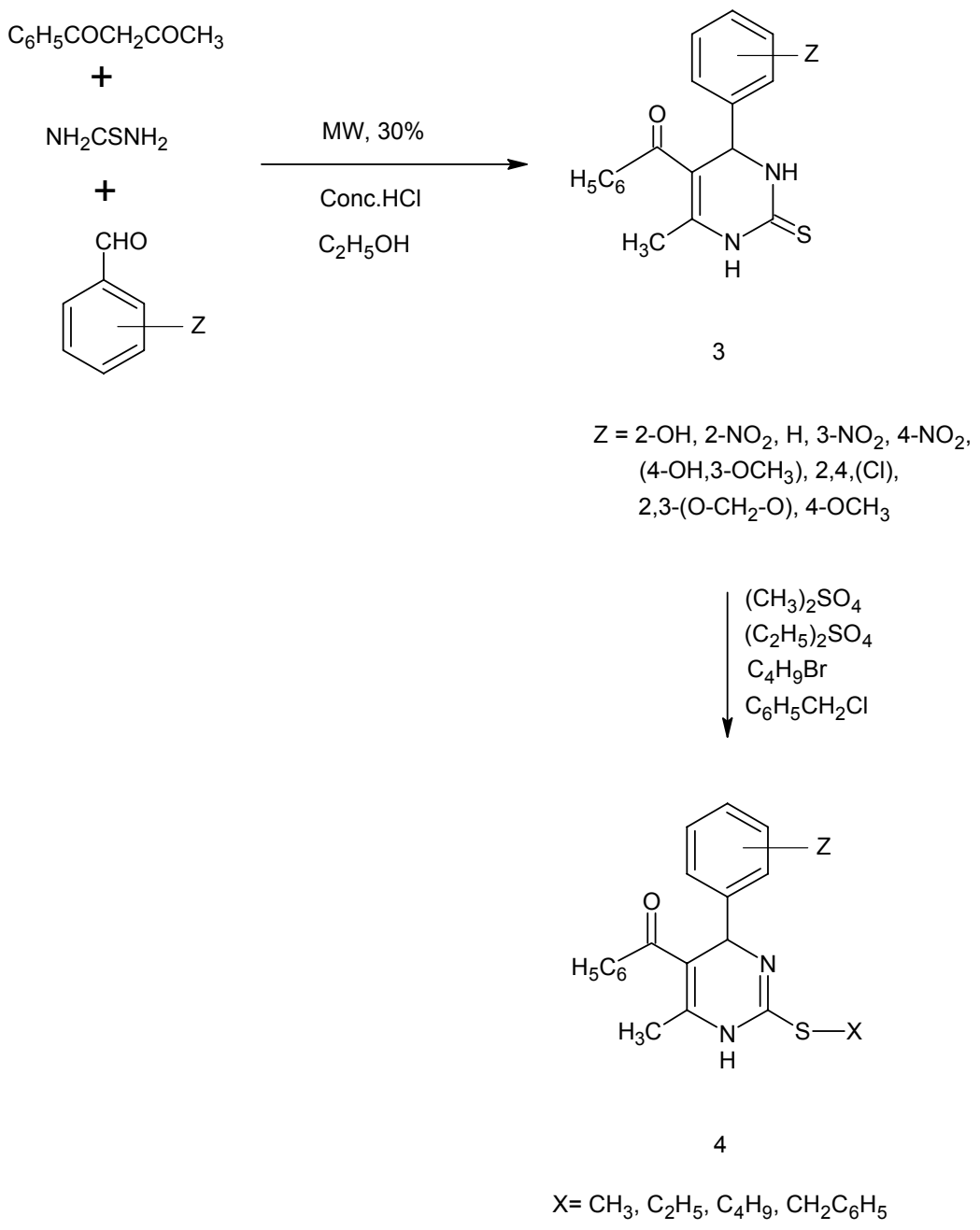

\section{Scheme 1}

Table 1. Synthesis of 5-benzoyl-6-methyl-4-substituted phenyl-2-thioxo-1,2,3,4tetrahydropyrimidine

\begin{tabular}{ccccc}
\hline 3a-i & $\mathrm{Z}$ & Time (minutes) & ${\mathrm{M}-\mathrm{P}^{0} \mathrm{C}}$ & \% yield \\
\hline $3 \mathrm{a}$ & $2-\mathrm{OH}$ & 4.0 & $240-241$ & 89 \\
$3 \mathrm{~b}$ & $2-\mathrm{NO}_{2}$ & 4.5 & $208-209$ & 75 \\
$3 \mathrm{c}$ & $\mathrm{H}$ & 5.0 & $228-230$ & 79 \\
$3 \mathrm{~d}$ & $4-\mathrm{OH}, 3-\mathrm{OCH}_{3}$ & 5.0 & $213-214$ & 82 \\
$3 \mathrm{e}$ & $3-\mathrm{NO}_{2}$ & 4.5 & $224-226$ & 78 \\
$3 \mathrm{f}$ & $4 \mathrm{NO}_{2}$ & 4.5 & $234-235$ & 80 \\
$3 \mathrm{~g}$ & $2,4-(\mathrm{Cl})$ & 5.0 & $204-205$ & 69 \\
$3 \mathrm{~h}$ & $2,3-\left(\mathrm{O}-\mathrm{CH}_{2}-\mathrm{O}\right)$ & 4 & $186-187$ & 70 \\
$3 \mathrm{i}$ & $4-\mathrm{OCH}_{3}$ & 4.5 & $170-172$ & 71 \\
\hline
\end{tabular}


Table 2. Synthesis of 5-benzoyl-6-methyl-4-substituted phenyl-2-S-alkyl-1, 4-dihydropyrimidine

\begin{tabular}{llllll}
\hline $4 \mathrm{a}-\mathrm{r}$ & $\mathrm{Z}$ & $\mathrm{X}$ & Time $(\mathrm{hrs})$ & $\mathrm{M}-\mathrm{P}^{0} \mathrm{C}$ & Yield \% \\
\hline $4 \mathrm{a}$ & $2-\mathrm{OH}$ & $\mathrm{CH}_{3}$ & 3.0 & $194-195$ & 65 \\
$4 \mathrm{~b}$ & $\mathrm{H}$ & $\mathrm{CH}_{3}$ & 3.0 & $151-152$ & 62 \\
$4 \mathrm{c}$ & $\mathrm{H}$ & $\mathrm{C}_{2} \mathrm{H}_{5}$ & 3.0 & $168-170$ & 67 \\
$4 \mathrm{~d}$ & $2-\mathrm{OH}$ & $\mathrm{C}_{2} \mathrm{H}_{5}$ & 3.0 & $159-160$ & 64 \\
$4 \mathrm{e}$ & $3-\mathrm{NO}_{2}$ & $\mathrm{C}_{2} \mathrm{H}_{5}$ & 3.0 & $109-110$ & 59 \\
$4 \mathrm{f}$ & $4-\mathrm{NO}_{2}$ & $\mathrm{C}_{2} \mathrm{H}_{5}$ & 3.0 & $118-120$ & 56 \\
$4 \mathrm{~g}$ & $2,4-(\mathrm{Cl})$ & $\mathrm{C}_{2} \mathrm{H}_{5}$ & 3.0 & $124-125$ & 52 \\
$4 \mathrm{~h}$ & $2-\mathrm{OH}$ & $\mathrm{CH}_{2}-\mathrm{C}_{6} \mathrm{H}_{5}$ & 5.0 & $152-154$ & 63 \\
$4 \mathrm{i}$ & $2,4-(\mathrm{Cl})$ & $\mathrm{CH}_{2}-\mathrm{C}_{6} \mathrm{H}_{5}$ & 5.0 & $182-183$ & 61 \\
$4 \mathrm{j}$ & $2-\mathrm{NO}{ }_{2}$ & $\mathrm{CH}_{2} \mathrm{C}_{6} \mathrm{H}_{5}$ & 5.0 & $168-169$ & 64 \\
$4 \mathrm{k}$ & $3-\mathrm{NO}_{2}$ & $\mathrm{CH}_{2}-\mathrm{C}_{6} \mathrm{H}_{5}$ & 5.0 & $205-207$ & 58 \\
41 & $4-\mathrm{NO}_{2}$ & $\mathrm{CH}_{2}-\mathrm{C}_{6} \mathrm{H}_{5}$ & 5.0 & $196-198$ & 56 \\
$4 \mathrm{~m}$ & $4-\mathrm{OH}$, & $\mathrm{CH}_{2}-\mathrm{C}_{6} \mathrm{H}_{5}$ & 5.0 & $161-162$ & 59 \\
& $3-\mathrm{OCH}$ & & & \\
$4 \mathrm{n}$ & $\mathrm{H}$ & $\mathrm{CH}_{2}-\mathrm{C}_{6} \mathrm{H}_{5}$ & 5.0 & $185-187$ & 58 \\
$4 \mathrm{o}$ & $4-\mathrm{NO}_{2}$ & $\mathrm{C}_{4} \mathrm{H}_{9}$ & 5.0 & $178-179$ & 55 \\
$4 \mathrm{p}$ & $3-\mathrm{NO}_{2}$ & $\mathrm{C}_{4} \mathrm{H}_{9}$ & 5.0 & $180-181$ & 57 \\
$4 \mathrm{q}$ & $2-\mathrm{OH}$ & $\mathrm{C}_{4} \mathrm{H}_{9}$ & 5.0 & $124-125$ & 61 \\
$4 \mathrm{r}$ & $\mathrm{H}$ & $\mathrm{C}_{4} \mathrm{H}_{9}$ & 5.0 & $190-192$ & 55 \\
\hline
\end{tabular}

\section{Experimental Section}

General Procedures. Melting points are uncorrected and were recorded in liquid paraffin-bath using open end capillaries. Thin layer chromatography was performed on Silicagel G (Merck). ${ }^{1} \mathrm{H}$ NMR spectra were recorded on AL-300F (Bruker), FTPMR Spectrometer. The IR spectra were recorded on Perkin-Elmer spectrum RX IFT-IR System. The mass spectra were obtained on a JEOL 5x102/DA-6000 mass spectrometer. All the compounds gave satisfactory elemental analysis within $\pm 0.4 \%$ of theoretical values. The microwave irradiated reactions were performed in domestic household microwave oven Samsung M1777N. Characterisation data of the compounds are given in table 3 . 
Table 3. Characterization data of the synthesized compounds

\begin{tabular}{|c|c|}
\hline Comp & ${ }^{1} \mathrm{H}$ NMR $(\delta, \mathrm{ppm})$; Mass \\
\hline \multirow[t]{3}{*}{$4 \mathbf{a}$} & $10.7(\mathrm{~s}, 1 \mathrm{H}, \mathrm{NH}), 10.5(\mathrm{~s}, 1 \mathrm{H},-\mathrm{OH}), 6.9-7.9(\mathrm{~m}, 9 \mathrm{H}, \mathrm{Ar}-\mathrm{H})$ \\
\hline & $4.2(\mathrm{~s}, 1 \mathrm{H}, 4-\mathrm{CH}), 2.5\left(\mathrm{~s}, 3 \mathrm{H}, \mathrm{S}-\mathrm{CH}_{3}\right), 1.8\left(\mathrm{~s}, 3 \mathrm{H}, 6-\mathrm{CH}_{3}\right)$ \\
\hline & $\mathrm{m} / \mathrm{z} 338(94.5 \%) \mathrm{M}^{+}, 321$ (40.8\%), 245 (30.6\%), $233(33.7 \%) 105(100 \%), 77$ (80.9\%) \\
\hline \multirow[t]{2}{*}{ 4b } & $\begin{array}{l}10.9(\mathrm{~s}, 1 \mathrm{H}, \mathrm{NH}), 7.3-7.6(\mathrm{~m}, 10 \mathrm{H}, \mathrm{Ar}-\mathrm{H}), 5.8(\mathrm{~s}, 1 \mathrm{H}, 4-\mathrm{CH}), 2.7\left(\mathrm{~s}, 3 \mathrm{H}, \mathrm{S}-\mathrm{CH}_{3}\right), 1.9(\mathrm{~s}, \\
\left.3 \mathrm{H}, 6-\mathrm{CH}_{3}\right)\end{array}$ \\
\hline & m/z 321 (40.8\%) M+, 245 (30.6\%), 130 (4.1\%), 115 (7.3\%), 105 (100\%), 77 (80.9\%) \\
\hline \multirow[t]{2}{*}{$4 \mathbf{c}$} & $\begin{array}{l}11.0(\mathrm{~s}, 1 \mathrm{H}, \mathrm{NH}), 7.3-7.6(\mathrm{~m}, 10 \mathrm{H}, \mathrm{Ar}-\mathrm{H}), 5.8(\mathrm{~s}, 1 \mathrm{H}, 4-\mathrm{CH}), 3.5(\mathrm{~m}, 1 \mathrm{H}, \mathrm{S}-\mathrm{CH}), 3.4(\mathrm{~m}, \\
1 \mathrm{H} . \mathrm{S}-\mathrm{CH}) 1.9\left(\mathrm{~s}, 3 \mathrm{H} .6-\mathrm{CH}_{3}\right), 1.2-1.3\left(\mathrm{t}, 3 \mathrm{H} . \mathrm{S}-\mathrm{CH}_{2}-\mathrm{CH}_{3}\right)\end{array}$ \\
\hline & $\begin{array}{l}\mathrm{m} / \mathrm{z} 334(100 \%) \mathrm{M}^{+}, 319(22.3 \%), 231(36.9 \%), 169(7.9 \%) ; 153(2.7 \%), 105(44.3 \%) \text {, } \\
77(43.0 \%)\end{array}$ \\
\hline \multirow[t]{2}{*}{ 4d } & $\begin{array}{l}10.8(\mathrm{~s}, 1 \mathrm{H}, \mathrm{NH}), 10.7(\mathrm{~s}, 1 \mathrm{H}, \mathrm{OH}), 6.9-8.0(\mathrm{~m}, 9 \mathrm{H}, \mathrm{Ar}-\mathrm{H}), 4.3(\mathrm{~s}, 1 \mathrm{H}, 4-\mathrm{CH}), 3.0-3.4 \\
\left(\mathrm{~m}, 2 \mathrm{H}, \mathrm{SCH}_{2}-\mathrm{CH}_{3}\right), 1.8\left(\mathrm{~s}, 3 \mathrm{H}, 6-\mathrm{CH}_{3}\right), 1.2\left(\mathrm{t}, 3 \mathrm{H}, \mathrm{S}_{-}-\mathrm{CH}_{2}-\mathrm{CH}_{3}\right)\end{array}$ \\
\hline & $\begin{array}{l}\mathrm{m} / \mathrm{z} 380(5.0 \%) \mathrm{M}^{+}, 363(2.3 \%), 352(48.5 \%), 335(30.6 \%), 323(20.4 \%), 275(5.0 \%) \text {, } \\
259(20.9 \%), 105(100 \%), 77(62.6 \%)\end{array}$ \\
\hline \multirow[t]{3}{*}{$4 \mathrm{~m}$} & $10.9(\mathrm{~s}, 1 \mathrm{H}, \mathrm{NH}), 8.1(\mathrm{~s}, 1 \mathrm{H}, \mathrm{OH}), 6.6-7.5(\mathrm{~m}, 13 \mathrm{H}, \mathrm{Ar}-\mathrm{H}), 5.7(\mathrm{~s}, 1 \mathrm{H}, 4-\mathrm{CH}), 4.9(\mathrm{~d}$ \\
\hline & $\left.1 \mathrm{H}, \mathrm{S}-\mathrm{CH}_{2}\right), 4.5\left(\mathrm{~d}, 1 \mathrm{H}, \mathrm{S}-\mathrm{CH}_{2}\right), 1.9\left(\mathrm{~s}, 3 \mathrm{H}, 6-\mathrm{CH}_{3}\right), 3.7\left(\mathrm{~s}, 3 \mathrm{H}, \mathrm{OCH}_{3}\right)$ \\
\hline & m/z 411 (0.93\%), 367 (2.67\%), 123 (6.0\%), 105 (9.2\%), 91 (100\%), 77 (22.7\%) \\
\hline $4 n$ & $\begin{array}{l}10.7(\mathrm{~s}, 1 \mathrm{H}, \mathrm{NH}), 6.4-7.2(\mathrm{~m}, 15 \mathrm{H} \mathrm{Ar}-\mathrm{H}), 5.1(\mathrm{~s}, 1 \mathrm{H}, 4-\mathrm{CH}), 4.6\left(\mathrm{~d}, 1 \mathrm{H}, \mathrm{S}-\mathrm{CH}_{2}\right), 4.2(\mathrm{~d}, \\
\left.1 \mathrm{H},-\mathrm{SCH}_{2}\right), 1.7\left(\mathrm{~s}, 3 \mathrm{H}, 6 \mathrm{CH}_{3}\right) \\
\mathrm{m} / \mathrm{z} 398 \mathrm{M}^{+}\end{array}$ \\
\hline 40 & $\begin{array}{l}10.8(\mathrm{~s}, 1 \mathrm{H}, \mathrm{NH}), 6.7-7.6(\mathrm{~m}, 9 \mathrm{H} \mathrm{Ar}-\mathrm{H}), 5.8(\mathrm{~s}, 1 \mathrm{H}, 4-\mathrm{CH}), 3.7\left(\mathrm{~m}, 1 \mathrm{H} \text { of } \mathrm{S}-\mathrm{CH}_{2}\right), 3.2 \\
\left(\mathrm{~m}, 1 \mathrm{H} \text { of } \mathrm{S}-\mathrm{CH}_{2}\right), 2.0\left(\mathrm{~s}, 3 \mathrm{H}, 6-\mathrm{CH}_{3}\right), 1.2-1.5\left(\mathrm{~m}, \mathrm{~S}-\mathrm{CH}_{2}-\mathrm{CH}_{2}-\mathrm{CH}_{2}-\mathrm{CH}_{3}\right), 0.8(\mathrm{t}, 3 \mathrm{H}, \\
\left.\mathrm{CH}_{3} \text { of S- } \mathrm{CH}_{2}-\mathrm{CH}_{2}-\mathrm{CH}_{2}-\mathrm{CH}_{3}\right) \\
\mathrm{m} / \mathrm{z} 409 \mathrm{M}^{+}\end{array}$ \\
\hline $4 \mathbf{r}$ & $\begin{array}{l}10.7(\mathrm{~s}, 1 \mathrm{H}, \mathrm{NH}), 7.3-7.5(\mathrm{~m}, 10 \mathrm{H}, \mathrm{Ar}-\mathrm{H}), 5.8(\mathrm{~s}, 1 \mathrm{H}, 4-\mathrm{CH}), 3.6(\mathrm{~m}, 1 \mathrm{H} \text { of S-CH}), 3.2 \\
\left(\mathrm{~m}, 1 \mathrm{H}, \mathrm{S}-\mathrm{CH}_{2}\right), 2.1\left(\mathrm{~s}, 3 \mathrm{H}, 6-\mathrm{CH}_{3}\right), 1.3-1.5\left(\mathrm{~m}, \mathrm{~S}-\mathrm{CH}_{2}-\mathrm{CH}_{2}-\mathrm{CH}_{2}-\mathrm{CH}_{3}\right), 0.8\left(\mathrm{t}, 3 \mathrm{H}, \mathrm{CH}_{3}\right. \\
\left.\text { of S-CH}-\mathrm{CH}_{2}-\mathrm{CH}_{2}-\mathrm{CH}_{3}\right) \\
\mathrm{m} / \mathrm{z} 364 \mathrm{M}^{+}\end{array}$ \\
\hline
\end{tabular}

General procedure for synthesis of 5-benzoyl-6-methyl-4-substituted phenyl-2-thioxo1,2,3,4-tetrahydropyrimidine

A mixture of benzoyl acetone $(0.015$ mole $2.4 \mathrm{~g})$, thiourea $(0.01 \mathrm{~mole}, 0.76 \mathrm{~g})$, and substituted aromatic aldehyde $(0.01$ mole) were subjected to microwave heating for 4-5 minutes using ethanol $(5 \mathrm{ml})$ as energy transfer medium and $\mathrm{HCl}(0.5 \mathrm{ml})$ as a catalyst. In most cases the reaction on long standing for 24-36 hrs, afforded product 3a-i which was filtered under reduced pressure and recrystallised out of methanol.

3(a) ${ }^{1} \mathrm{H}$ NMR $\left(300 \mathrm{MHz} \mathrm{CDCl}_{3}+\mathrm{DMSO}\right): \delta 8.8(\mathrm{~s}, 1 \mathrm{H}, \mathrm{NH}), 8.5(\mathrm{~s}, 1 \mathrm{H}, \mathrm{NH}), 6.8-7.9(\mathrm{~m}, 9 \mathrm{H}$, Ar-H), $4.5(\mathrm{~s}, 1 \mathrm{H},-\mathrm{OH}), 4.1(\mathrm{~s}, 1 \mathrm{H}, 4-\mathrm{CH}), 1.8\left(\mathrm{~s}, 3 \mathrm{H}, 6-\mathrm{CH}_{3}\right)$ Mass fragments m/z : $324(100 \%$ 
$\mathrm{M}^{+}$), 309 (60\%), 307 (21\%), 231 (17\%), $219(40 \%), 105(91 \%)$ and $77(60 \%) . \quad$ IR (Nujol) $\mathrm{cm}^{-}$ ${ }^{1}$ : 3380 (O-H str.), 3208 (sec. N-H str.) 3071 (aromatic C-H str.), 1669 (C=O str.) 1547 (C==C str.), 1449 ( $\mathrm{C}=\mathrm{C}$ str.), 1224 ( $\mathrm{C}=\mathrm{S}$ str.).

General procedure for synthesis of 5-benzoyl-6-methyl-4-substituted phenyl-2-Smethyl/ethyl-1, 4-dihydropyrimidine

To tetrahydropyrimidine ( 0.004 mole) 3 dissolved in methanol was added $\mathrm{NaOH}$ solution which was prepared by dissolving $\mathrm{NaOH}(0.160 \mathrm{~g})$ in water $(2 \mathrm{ml})$. The mixture was cooled. To this mixture dimethyl sulphate $(0.004$ mole, $0.5 \mathrm{ml})$ or diethyl sulphate $(0.004$ mole, $0.6 \mathrm{ml})$ was added dropwise while stirring the reaction mixture continuously. Then the reaction mixture was refluxed for $3 \mathrm{hrs}$. The reaction mixture was cooled and poured over crushed ice. Solid separated was filtered under reduced pressure, dried and recrystallised from methanol to give $4 \mathrm{a}-\mathrm{g}$. Spectral data are given in Table 3.

\section{General procedure for synthesis of 5-benzoyl-6-methyl-4-substituted phenyl-2-S-benzyl-1,} 4-dihydropyrimidine

To tetrahydropyrimidine $3(0.004$ mole $)$ dissolved in alcohol $(5 \mathrm{ml})$ was added benzyl chloride $(0.5 \mathrm{ml}, 0.004$ mole $)$ and the reaction mixture was refluxed for $5 \mathrm{hrs}$. The mixture was cooled at room temperature. The solid separated was filtered and recrystallised from ethanol to give 4h-n. Spectral data given in Table 3.

\section{General procedure for synthesis of 5-benzoyl-6-methyl-4-substituted phenyl-2-S-butyl-1, 4- dihydropyrimidine}

A mixture of powdered tetrahydropyrimidine 3 ( 0.004 mole), butyl bromide $(0.5 \mathrm{ml}, 0.004$ mole $)$ and absolute alcohol $(5 \mathrm{ml})$ was refluxed for $5 \mathrm{hrs}$. Then the product was allowed to separate at room temperature. After a long standing of 36-40 hrs., the product separated was filtered under reduced pressure and recrystallised from methanol to give 4o-r. Spectral data is given in Table 3.

\section{Biological activity}

To study the biological activity, experiments were conducted on rat uterus and rabbit heart. Compounds $4 \mathrm{~b}, 4 \mathrm{j}$ and $4 \mathrm{q}$ were screened for calcium channel blocking activity. Nifedipine was used as a standard drug for comparison. The activity is reported by measuring $\mathrm{IC}_{50}$ (inhibitory concentration, $\mu \mathrm{g} / \mathrm{mL}$ ) values of these compounds on female albino rat uterus. The results are presented in Table 4.

Table 4. Antihypertensive activity of synthesized compounds

\begin{tabular}{|c|c|}
\hline Comp. & $\begin{array}{c}\mathrm{IC}_{50} \text { (Inhibitory concentration } \\
\mu \mathrm{g} / \mathrm{mL})\end{array}$ \\
\hline $4 b$ & $17 \mu \mathrm{g} / \mathrm{ml}$ \\
\hline $4 j$ & $22 \mu \mathrm{g} / \mathrm{ml}$ \\
\hline $4 q$ & $28 \mu \mathrm{g} / \mathrm{ml}$ \\
\hline
\end{tabular}


Potent $\mathrm{Ca}^{2+}$ channel blocking activity was found in this series of compounds-S-methyl derivative is the most potent calcium channel blocker as it has lowest $\mathrm{IC}_{50}$ value as is evident from Table 4.

In rabbit heart as these compounds cause increase in coronary flow as well as increase in amplitude. These compounds can be useful in conditions like congestive heart failure (CHF) while other calcium channel blockers like nifedipine decrease the force of contraction of heart so cannot be useful in such conditions. The same property appears in commonly known drug digoxin.

\section{Conclusions}

On comparing S-methyl derivative of 1, 4-dihydropyrimidine with nifedipine, it has been found that it is most potent and S-methyl moiety is present on right side of the boat shaped conformation. This shows that one cannot ignore the structural details at right hand side as reported earlier.

Substitution with 2- $\mathrm{NO}_{2}, 2-\mathrm{OH}$ in aromatic ring also changes biological activity.

When benzoyl group was substituted at position 5; it increased the force of contraction of heart, which made these compounds useful in congestive heart failure conditions.

\section{Acknowledgements}

We are thankful to Department of Pharmacology, Govt. Medical College, Patiala - 147 002, Punjab (India) for evaluating Biological activities.

\section{References}

1. Sircar, I.; Gregor, E. K.; Anderson, K. R.; Haleen, S. J.; Shih, Y. H.; Weishaar, R. E.; Steffen, R. P.; Pugsley, T. A.; Taylor, M. D. J. Med. Chem. 1991, 34, 2249.

2. Guarneri, L., Patrizia, A., Marina, I., Elena, P., Carlo, T., Amedeo, L.; Rodolfo, T. Arzneimittel Forschung 1996, 46(10), 15; Biol. Abstr. 1998, 101 (9), 32842.

3. Wetzel, J. M.; Miao, S. W.; Borden, L. A.; Branhek, T. A.; Gluchowski, C. J. Med. Chem. 1995, 38, 1579.

4. Sweet, F.; Fissekis, J. D. J. Am. Chem. Soc. 1973, 95, 8741.

5. Janis, R. A.; Silver, P. J.; Triggle, J. Adv. Drug Res. 1987, 16, 309.

6. Bossert, F.; Vater, W. Med. Res. Rev. 1989, 9, 291.

7. Atwal, K. S.; Rovnyk, G. C.; Kimball, S. D.; Floyd, D. M.; Moreland, S. J. Med. Chem. 1990, 33, 2629. 
8. Rovnyk, G. C.; Atwal, K. S.; Kimball, S. D. J. Med. Chem. 1992, 35, 3254.

9. Fossheim, R.; Svarteng, K.; Mostad, A.; Romming, C.; Shefter, E.; Triggle, D. J. J. Med. Chem. 1982, 25, 125.

10. Rovnyak, G. C.; Kimball, S. D.; Beyer, B.; Gougoutas, P. J. Med. Chem. 1995, 38, 119.

11. Rana, K.; Kaur, B.; Kumar, B. Ind. J. Chem. 2004, 43B, 1553.

12. Chari, M. A.; Shobha, D.; Kumar, T. K.; Dubey, P. K. Arkivoc 2005, (xv), 74.

13. Kaur, B.; Kumar, B.; Kaur, J. Chem. Environ. Res. 2002, 11(3\&4), 203. 